\title{
Lung Microvascular and Arterial Endothelial Cells Differ in Their Responses to Intercellular Adhesion Molecule-1 Ligation
}

\author{
Qin Wang, Gordon R. Pfeiffer II, Troy Stevens, and Claire M. Doerschuk \\ Division of Integrative Biology, Department of Pediatrics, Rainbow Babies and Children's Hospital and Case Western Reserve University, \\ Cleveland, Ohio; and Department of Pharmacology, University of South Alabama, Mobile, Alabama
}

\begin{abstract}
Neutrophil adherence to tumor necrosis factor- $\alpha$ (TNF- $\alpha$ )-treated human pulmonary microvascular endothelial cells (PMECs) induces cytoskeletal changes in endothelial cells that require intercellular adhesion molecule-1 (ICAM-1)-dependent signaling events. This study determined whether similar changes occurred in rat PMECs and whether rat pulmonary arterial endothelial cells (PAECs) responded differently. Neutrophil adherence induced an increase in the formation of F-actin and in the apparent stiffness of TNF- $\alpha-$ treated rat PMECs. These responses, however, were absent in PAECs. To determine the mechanisms underlying these differences, ICAM-1-mediated signaling events were compared. Upregulation of ICAM- 1 by TNF- $\alpha$ and redistribution of ICAM- 1 induced by crosslinking antibodies were similar in both cell types. However, neutrophil adherence induced production of reactive oxygen species only in PMECs and not in PAECs. Moreover, phosphorylation of p38 mitogen-activated protein kinase induced by ICAM-1 cross-linking occurred only in PMECs and not in PAECs. This increase in p38 phosphorylation in PMECs was inhibited by allopurinol, a xanthine oxidase inhibitor. These data demonstrated that whereas TNF- $\alpha$ upregulated ICAM-1 and ICAM-1 cross-linking induced a similar redistribution of ICAM-1 on the endothelial cell surface, ICAM-1 ligation initiated p38 activation and cytoskeletal rearrangements only in PMECs and not in PAECs. Thus, neutrophil adhesion through ICAM-1 induced signaling events leading to cytoskeletal changes only in PMECs, the site of neutrophil emigration and edema formation, and not in PAECs.
\end{abstract}

Keywords: intercellular adhesion molecule-1; signaling; lung; endothelial cell

Neutrophil adhesion to pulmonary capillary endothelial cells (ECs) is thought to be required for neutrophil emigration during many pulmonary inflammatory responses. The mechanisms regulating neutrophil migration along ECs to EC borders after adhesion are not clearly defined. Recent studies using cultured human pulmonary microvascular endothelial cells (PMECs) demonstrate that neutrophil adherence to tumor necrosis factor (TNF)- $\alpha$-treated ECs induces changes in the F-actin cytoskeleton in ECs, as indicated by an increase in the apparent stiffness of ECs and in the formation of F-actin in ECs $(1,2)$. These changes in the EC cytoskeleton depend on intercellular adhesion molecule-1 (ICAM-1)-

(Received in original form January 7, 2002; accepted in final form June 20, 2002) Supported by NIH HL 48160, HL 33009, and a Clinical Scientist Award in Translational Research from the Burroughs Wellcome Fund (C.M.D.) and NHLBI F32 HL10177-01, a research grant from the American Lung Association, and Parker B. Francis Fellowship from the Francis Families Foundation (Q.W.).

Correspondence and requests for reprints should be addressed to Claire $M$. Doerschuk, M.D., Rainbow Babies and Children's Hospital and Case Western Reserve University, Room 787, 11100 Euclid Avenue, Cleveland, $\mathrm{OH} 44106$. E-mail: cmd22@po.cwru.edu

Am J Respir Crit Care Med Vol 166. pp 872-877, 2002

DOI: $10.1164 /$ rccm.2201007

Internet address: www.atsjournals.org induced signaling events. Ligation of ICAM-1 by neutrophils or cross-linking antibodies initiates production of reactive oxygen species (ROS) and subsequent activation of p38 mitogen-activated protein kinase (MAPK) $(1,3)$. Activation of $\mathrm{p} 38$, in turn, induces phosphorylation of heat shock protein 27 , an actin-binding protein that may mediate actin polymerization upon phosphorylation, and is required for the EC stiffening response as well as for neutrophil migration toward EC borders (3). These studies suggest that ICAM-1-dependent activation of ECs induced by neutrophil adherence plays important roles in modulating neutrophil migration toward EC borders.

In culture, rat PMECs form tighter junctions than do pulmonary arterial endothelial cells (PAECs), and only PAECs respond to calcium ionophores by increasing their permeability (4), suggesting that there may be site-specific differences in the response of ECs during inflammation. The hypothesis of the present study is that ICAM-1-mediated signaling events that are initiated by neutrophil adhesion are different in PAECs compared with PMECs, the site of neutrophil emigration and edema formation. This study compared the cytoskeletal changes in response to neutrophil adherence in PMECs and PAECs isolated from rats. These results led to studies comparing ICAM-1-initiated signaling events, including ICAM-1 clustering, activation of xanthine oxidase, production of intracellular oxidants, and activation of p38 MAPK.

\section{METHODS}

\section{Rat Neutrophil Isolation}

Rat neutrophils were isolated from venous blood using neutrophil isolation medium- 2 density gradients according to the manufacturer's protocols, with purity higher than 95\% (Cardinal Associates, Santa Fe, NM).

\section{Isolation and Cultivation of Rat PAECs and PMECs}

Rat PAECs were obtained by gently scraping the lumenal surface of isolated rat pulmonary arteries, and their EC identity was verified as described previously $(5,6)$. Rat PMECs were isolated and cultured using a modified method described previously (7).

\section{Measurement of ICAM-1 Expression}

The expression of ICAM-1 was quantified by ELISA as described previously (1). ECs were incubated with $10 \mu \mathrm{g} / \mathrm{ml}$ anti-ICAM-1 (clone 1A29; PharMingen, San Diego, CA) or control antibody for 1 hour followed by incubation with $10 \mu \mathrm{g} / \mathrm{ml}$ fluorescein-conjugated secondary antibody for 1 hour. The fluorescence was quantified using a fluorescent plate reader. After subtracting background fluorescence, the fold changes in ICAM-1 expression after TNF- $\alpha$ treatment compared with the untreated control cells (time 0 ) were calculated:

Fold change at time $\mathrm{X}=$ the fluorescence intensity at time $\mathrm{X} /$ the average fluorescence intensity at time 0 .

\section{Biomechanical Properties of ECs Evaluated Using Magnetic Twisting Cytometry}

The biomechanical properties of ECs were measured using magnetic twisting cytometry as described previously (1-3). ECs were incubated 
with anti- $\beta_{1}$-integrin antibody-coated ferromagnetic beads and placed in the magnetic twisting cytometer. After measuring the baseline stiffness of ECs, isolated rat neutrophils were added to ECs (ratio $=1: 1$ ), and the apparent stiffness of ECs was measured 2-15 minutes later.

\section{F-actin Visualization and Quantification}

F-actin distribution in ECs was visualized using rhodamine phalloidin stain as described previously (2).

\section{Evaluation of Xanthine Oxidase Activity in ECs upon Neutrophil Adherence}

The activity of xanthine oxidase in ECs was evaluated by native gel electrophoresis followed by enzymatic detection (10). ECs treated for 24 hours with $20 \mathrm{ng} / \mathrm{ml} \mathrm{TNF}-\alpha$ were washed, and rat neutrophils were added (neutrophil:EC = 1:1) for 2-15 minutes. The cells were washed and lysed in buffer containing $0.2 \%$ Triton X-100, $50 \mathrm{mM}$ potassium phosphate, $100 \mathrm{mM}$ iodoacetic acid, $2 \mathrm{mM}$ DTT, and a cocktail of protease inhibitors. The cell lysates were centrifuged at $3000 \times g$ at $4^{\circ} \mathrm{C}$ for 10 minutes, and $150 \mu \mathrm{g}$ protein/lane was subjected to gel electrophoresis using native $8 \%$ polyacrylamide Tris-glycine gels under nonreducing conditions. Purified xanthine oxidase isolated from buttermilk was used as the positive control. The gels were stained in $50 \mathrm{mM}$ Tris- $\mathrm{HCl}$ containing $1.4 \mathrm{mM}$ hypoxanthine and $0.25 \mathrm{mM}$ nitro blue tetrazolium as described (8).

\section{Measurement of Oxidant Production in ECs}

Oxidant production in ECs in response to neutrophil adhesion was evaluated by measuring changes in $2^{\prime}, 7^{\prime}$-dichlorofluorescein (DCF) fluorescence as described (1).

\section{ICAM-1 Cross-Linking}

ICAM-1 cross-linking in ECs treated for 24 hours with $20 \mathrm{ng} / \mathrm{ml} \mathrm{rat}$ TNF- $\alpha$ was performed as described $(1,9)$.

\section{Visualization of ICAM-1 Distribution}

ICAM-1 distribution before or after cross-linking was visualized as described $(3,10)$.

\section{Detection of p38 MAPK Phosphorylation}

Total p38 MAPK and the phosphorylated p38 MAPK were detected by immunoblot as described (3).

\section{Statistical Analysis}

Data were analyzed using the Student's $t$ test or one-way analysis of variance. A $p$ value less than 0.05 was considered significant. The data are expressed as the mean value \pm SEM.

\section{RESULTS}

\section{Neutrophil Adherence-induced Cytoskeletal Changes in TNF- $\alpha$-treated PMECs and PAECs}

Changes in the actin cytoskeleton in TNF- $\alpha$-treated ECs in response to neutrophil adherence were evaluated by magnetic twisting cytometry using ferromagnetic beads coated with anti- $\beta 1$-integrin antibody $(1,2)$. In TNF- $\alpha$-treated rat PMECs, neutrophil adherence for 2-15 minutes increased the apparent stiffness of ECs (Figure 1A). Addition of buffer rather than neutrophils had no effect on the apparent stiffness of ECs. This increase in the apparent stiffness of ECs required pretreatment with TNF- $\alpha$ because addition of neutrophils to untreated ECs had no effect on the apparent stiffness of ECs (data not shown). These observations were similar to those made using cultured human PMECs $(1,2)$. In striking contrast, the apparent stiffness of PAECs was less than half that of PMECs, and neutrophil adherence to PAECs for 2-15 minutes did not increase their apparent stiffness (Figure 1B).
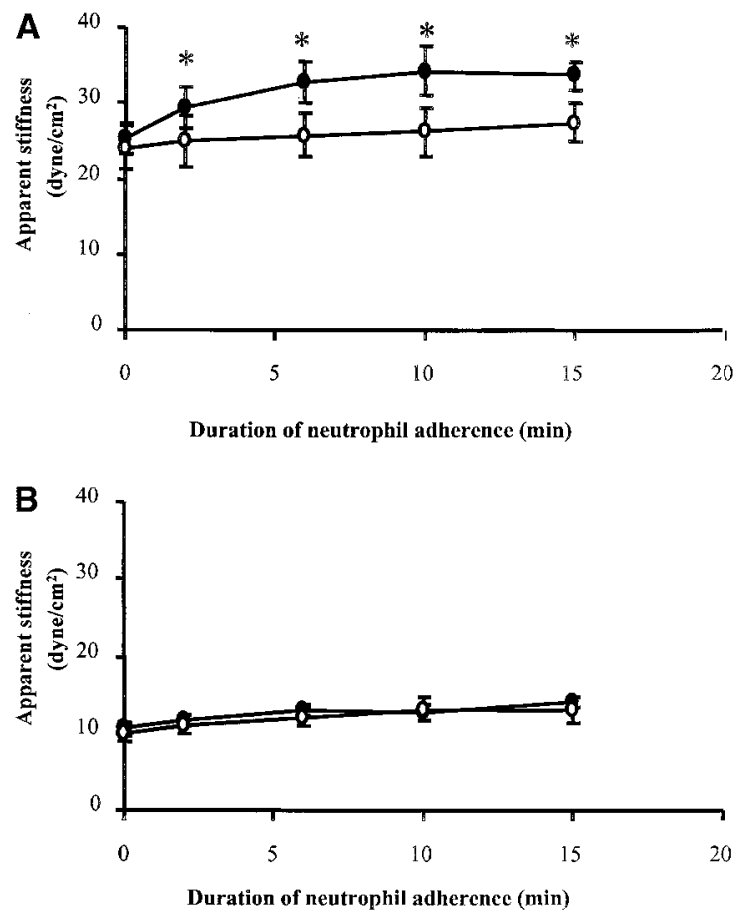

Figure 1. Changes in rat EC stiffness induced by neutrophil adherence. After TNF- $\alpha$ was washed off, the apparent stiffness of TNF- $\alpha$-treated PMECs $(A)$ or PAECs $(B)$ was measured by a magnetic twisting cytometer using ferromagnetic beads bound to $\beta_{1}$-integrin (see METHODS). After the measurement of baseline stiffness, either purified neutrophils (neutrophil:EC $=1: 1$ ) or buffer was added to the well, and EC stiffness was measured $2,6,10$, and 15 minutes later. The data are expressed as mean $\pm \operatorname{SEM}(n=4) .{ }^{*} p<0.05$ when compared with the baseline stiffness. Closed circles, addition of neutrophils; open circles, addition of buffer.

To further evaluate the cytoskeletal changes in these two types of ECs in response to neutrophil adherence, the average staining intensity of F-actin in TNF- $\alpha$-treated ECs was measured. Neutrophil adherence for 2-10 minutes induced a significant increase in the F-actin staining in PMECs but not in PAECs (Figure 2). This difference was not due to less neutrophil adhesion because neutrophils adhere similarly to TNF- $\alpha$-treated PMECs and PAECs. After 10 minutes of adhesion, the number of neutrophils adherent to TNF- $\alpha$-treated PMECs and PAECs was $6.6 \pm 1.0$ and $6.5 \pm 1.1$ per $\times 600$ field, respectively. Taken together, these studies demonstrate that neutrophil adherence induces cytoskeletal responses only in PMECs and not in PAECs.

ICAM-1 Expression Induced by TNF- $\alpha$ and ICAM-1 Redistribution Induced by Cross-Linking Antibodies in PMECs and PAECs

Previous studies demonstrated that neutrophil adherenceinduced cytoskeletal changes in TNF- $\alpha$-treated human PMECs require ICAM-1-mediated signaling events $(1,3)$. The expression of ICAM-1 in response to TNF- $\alpha$ treatment was therefore examined to determine whether the different responses elicited by neutrophils in PAECs and PMECs were due to differences in ICAM-1 expression. Treatment with TNF- $\alpha$ for 4 or 24 hours induced a significant increase in ICAM-1 expression in both cell types, and the increase was similar in these two cell types (Figure 3).

Previous studies demonstrated that ICAM-1-induced signaling events require cross-linking with a secondary antibody, sug- 


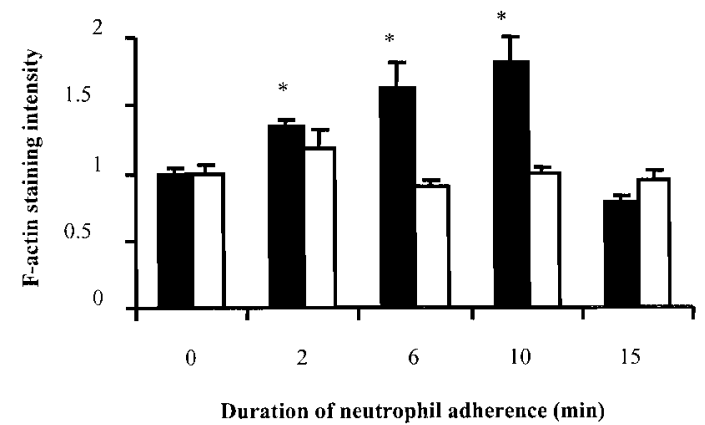

Figure 2. Quantification of F-actin staining in TNF- $\alpha$-activated rat ECs upon neutrophil adherence. Rat ECs were treated with TNF- $\alpha$ and washed. Purified rat neutrophils were added to ECs (neutrophil:EC = 1:1) and allowed to adhere for 2-15 minutes. F-actin was detected by rhodamine-phalloidin stain (see METHODS), and the image was scanned and monitored using a laser scanning confocal microscope. The mean pixel intensity of the F-actin fluorescence per field $(\times 1,000)$ occupied by F-actin staining was measured. The data are normalized to the values of the control cells without neutrophil adhesion and are presented as mean \pm SEM from at least four images for each slide. The experiment was repeated two times with similar results. ${ }^{*} \mathrm{p}<0.05$ when compared with controls without neutrophil adhesion. Closed bars, PMECs; open bars, PAECs.

gesting that ICAM-1 clustering may be required (1, 3). ICAM-1 distribution with or without the addition of a cross-linking secondary antibody in TNF- $\alpha$-treated PMECs and PAECs was then examined. The addition of a secondary antibody induced ICAM-1 redistribution and formation of ICAM-1 aggregates similarly in both cell types (Figure 4). These data demonstrate that ICAM-1 clustering in response to a cross-linking antibody is not differentially regulated in these cell types.

\section{ICAM-1-Dependent Signaling Events in TNF- $\alpha$-treated PMECs and PAECs: Activation of Xanthine Oxidase, Generation of ROS, and Activation of p38 MAPK}

Previous studies demonstrated that ICAM-1-dependent, allopurinol-inhibitable production of ROS and subsequent activation of p38 MAPK are required for the cytoskeletal changes in human PMECs induced by neutrophil adherence (3). Because neutrophil-induced EC stiffening and cytoskeletal changes only occur in rat PMECs, these signaling pathways induced by ICAM-1 ligation in PMECs and PAECs were compared.

The activity of xanthine oxidase was examined by in-gel activ-

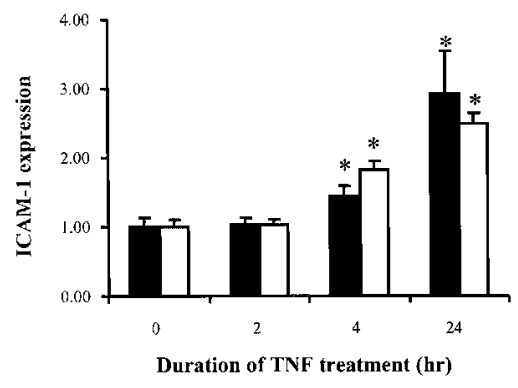

Figure 3. Changes in ICAM-1 expression in rat pulmonary ECs in response to TNF- $\alpha$ treatment. Rat PAECs and PMECs were treated with $20 \mathrm{ng} / \mathrm{ml}$ TNF- $\alpha$ for 4 or 24 hours, and ICAM-1 expression in ECs was quantified by ELISA (see Methods). The raw fluorescence values for PAECs and PMECs not treated with TNF- $\alpha$ measured 2,007 \pm 182 and 2,891 \pm 348 , respectively $(n=8)$. Data were expressed as fold changes compared with cells not treated with TNF- $\alpha$ and are presented as mean \pm SEM $(n=8) .{ }^{*} p<0.05$ when compared with cells not treated with TNF- $\alpha$. Closed bars, PMECs; open bars, PAECs.
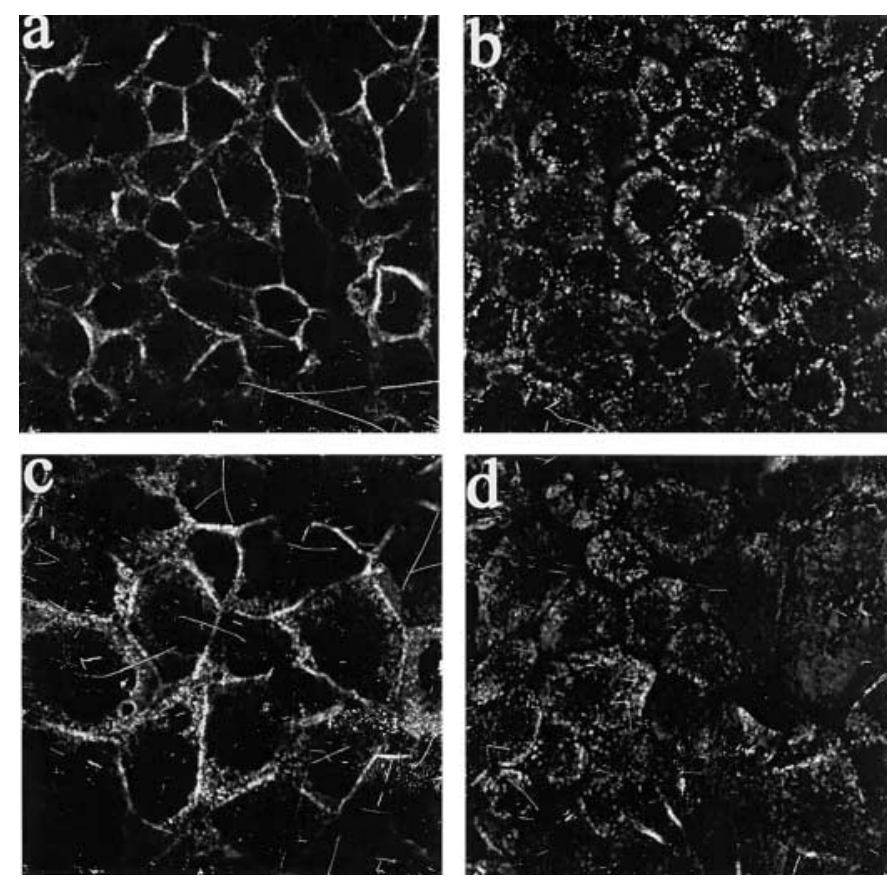

Figure 4. ICAM-1 redistribution induced by a cross-linking secondary antibody in rat ECs. TNF- $\alpha$-treated PAECs $(A, B)$ or PMECs $(C, D)$ were incubated with $15 \mu \mathrm{g} / \mathrm{ml} \mathrm{ICAM}-1$ antibody for 30 minutes and washed. The cells were either fixed and incubated with fluorescein-conjugated goat anti-mouse lgG for 15 minutes $(A, C)$ or incubated with fluoresceinconjugated goat anti-mouse $\lg G$ for 15 minutes $(B, D)$ and then fixed.

ity stain, using hypoxanthine as the substrate (Figure 5). A standard curve was constructed using purified xanthine oxidase at different concentrations (Figure 5A). For each experiment, purified xanthine oxidase $(0.132 \mu \mathrm{U})$ was used as a standard to calculate the activity of xanthine oxidase in the samples. Cell lysates containing $100-150 \mu \mathrm{g}$ proteins were used to measure xanthine oxidase activity in PAECs and PMECs. The densitometric values were always less than one and were therefore located on the region of the standard curve that fits all the data points (Figures 5A and 5B). Treatment with TNF- $\alpha$ for 24 hours did not alter the activity of xanthine oxidase in either cell type (data not shown). As shown in Figure 5, the baseline activity of xanthine oxidase (expressed as $\mu \mathrm{U} / \mathrm{mg}$ protein) in TNF- $\alpha-$ pretreated PAECs was significantly higher than that in PMECs $(0.65 \pm 0.06$ versus $0.18 \pm 0.02 \mu \mathrm{U} / \mathrm{mg}$ protein, $\mathrm{n}=6-8, \mathrm{p}<$ $0.05)$. In response to neutrophil adherence for up to 15 minutes, no significant increase in xanthine oxidase activity was observed in PAECs $(\mathrm{p}>0.05, \mathrm{n}=4)$. In PMECs, neutrophil adherence induced an increase within 15 minutes (from $0.18 \pm 0.02$ to $0.33 \pm 0.06 \mu \mathrm{U} / \mathrm{mg}$ protein, $\mathrm{p}<0.05, \mathrm{n}=5$ ) (Figures $5 \mathrm{~B}$ and $5 \mathrm{C})$. Because neutrophils can be stimulated to release elastase on exposure to chemoattractants (10) and this enzyme may cleave xanthine dehydrogenase to the active oxidase, the effect of exogenous leukocyte elastase on EC xanthine oxidase activity was examined. Incubating either EC type with $0.1 \mathrm{U}$ exogenous leukocyte elastase for 15 minutes did not induce an increase in xanthine oxidase activity (data not shown). In addition, mixing EC lysates with an equal number of neutrophils did not increase xanthine oxidase activity (data not shown).

This observed difference in xanthine oxidase activity in PAECs and PMECs, both basally and that induced by neutrophil adherence, led us to examine the production of ROS in ECs by measuring the changes in DCF fluorescence in response to 

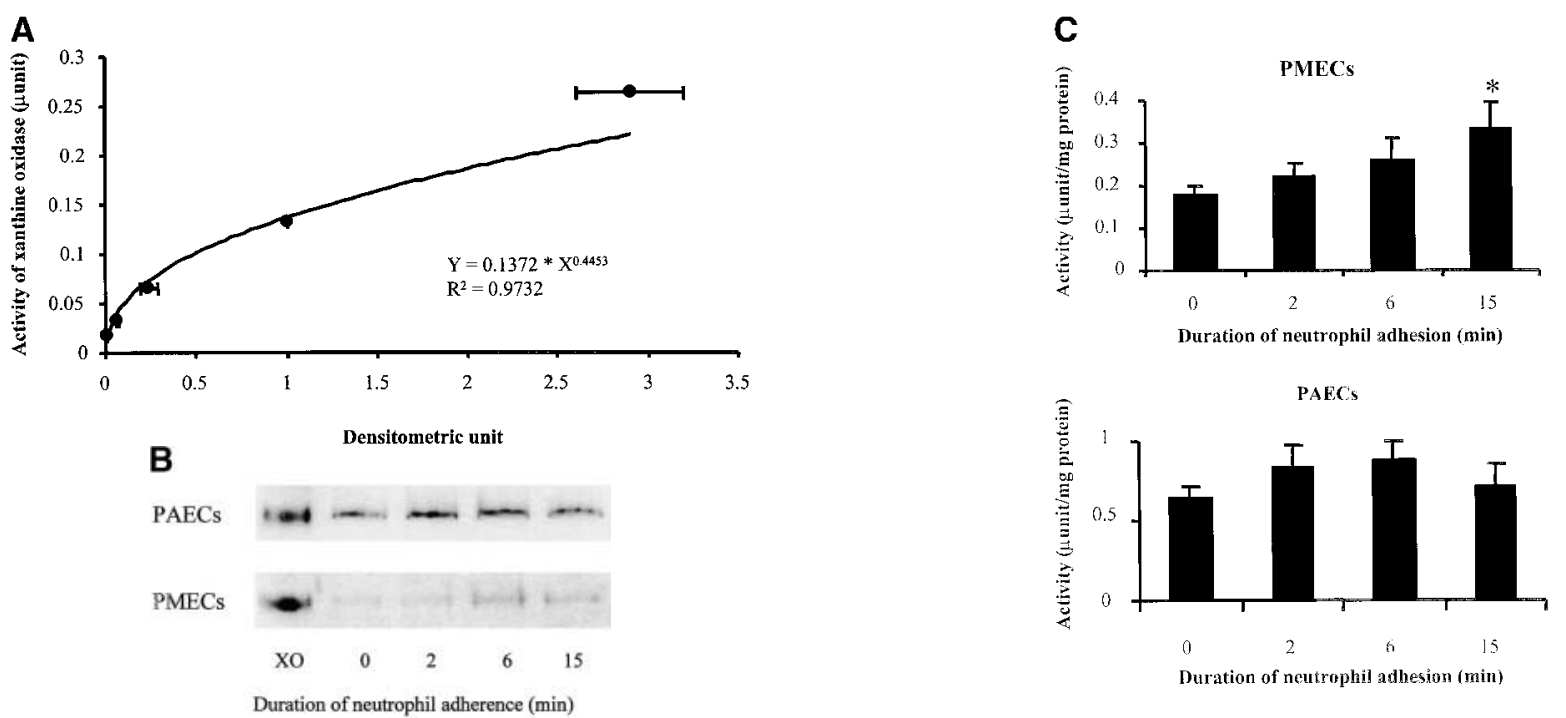

Figure 5. Xanthine oxidase activity in rat ECs in response to neutrophil adherence. TNF- $\alpha$-treated PAECs or PMECs were washed twice with cultured medium. Purified rat neutrophils were added to ECs (neutrophil:EC =1:1) and were allowed to adhere for 2, 6, and 15 minutes. Xanthine oxidase activity was determined as described in METHODS. ( $A$ ) Standard curve of xanthine oxidase activity. Purified xanthine oxidase at different concentrations was used to construct the standard curve $(n=4)$. $(B)$ Representative gel images showing xanthine oxidase activity in PAECs or PMECs before or after neutrophil adhesion. Purified xanthine oxidase $(0.132 \mu \mathrm{U})$ was used as the standard, and cell lysates containing $150 \mu \mathrm{g}$ proteins were used for each sample. (C) Activity of xanthine oxidase in PMECs or PAECs on neutrophil adhesion. The activity was calculated on the basis of the standard curve and the standard used in each experiment. The data are presented as microunits per milligram of protein and are represented as mean $\pm \operatorname{SEM}(n \geqslant 4)$.

addition of neutrophils or buffer. In the absence of neutrophil adherence, neither the baseline DCF fluorescence nor the rate of changes in DCF fluorescence was different in PAECs and PMECs (Figure 6). In PMECs, addition of neutrophils induced a time-dependent increase in DCF fluorescence compared with the addition of buffer, indicating that ROS production in ECs occurred in response to neutrophil adherence (Figure 6A). In contrast, no increase in DCF fluorescence was observed in PAECs when neutrophils adhered (Figure 6B).

Because p38 MAPK is a downstream target of ROS production in human PMECs on ICAM-1 ligation (3), this difference in ROS production in PAECs and PMECs led us to examine p38 activity in response to ICAM-1 cross-linking using an antibody that recognizes the diphosphorylated form of p38 MAPK (activated p38). Cross-linking ICAM-1 with a secondary antibody for 2-15 minutes induced a significant increase in p38 phosphorylation only in PMECs and not in PAECs (Figures 7A and $7 \mathrm{~B})$.

To determine whether activation of xanthine oxidase was required for $\mathrm{p} 38$ phosphorylation in rat PMECs, cells were pretreated with allopurinol, a xanthine oxidase inhibitor. In cells pretreated with control vehicle, ICAM-1 cross-linking for 6 minutes induced a significant increase in p38 phosphorylation (Figure 8). This increase was completely inhibited by allopurinol. These data, together with our previous observations (3), demonstrate that p38 activation in response to ICAM-1 cross-linking is ROS dependent in both rat and human PMECs.

\section{DISCUSSION}

This study demonstrated that neutrophil adherence-induced cytoskeletal changes occurred only in TNF- $\alpha$-pretreated PMECs and not in PAECs. Neutrophil adherence-induced EC stiffening response and increases in $\mathrm{F}$-actin formation were observed only in TNF- $\alpha$-pretreated PMECs, the site of neutrophil emigration and edema formation during pulmonary inflammation. Subse-
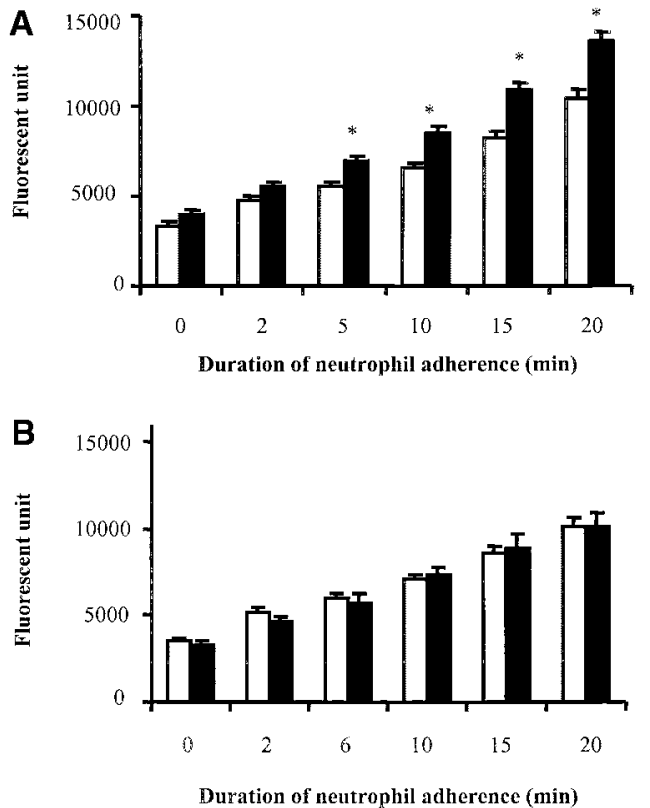

Figure 6. Oxidant production in rat ECs in response to neutrophil adherence. TNF- $\alpha$-activated rat ECs were loaded with $2^{\prime}, 7^{\prime}$-dichlorofluorescein diacetate and washed. After measuring the baseline DCF fluorescence, neutrophils or buffer was added, and the DCF fluorescence was measured 2-20 minutes later. The data are presented as DCF fluorescence units before or after the addition of neutrophils or buffer. $(A)$ Changes in DCF fluorescence units in PMECs. (B) Changes in DCF fluorescence units in PAECs. The data are expressed as mean \pm SEM of eight wells. ${ }^{*} \mathrm{p}<0.05$ when compared with the corresponding control cells. Open bars, addition of buffer; closed bars, addition of neutrophils. 
A

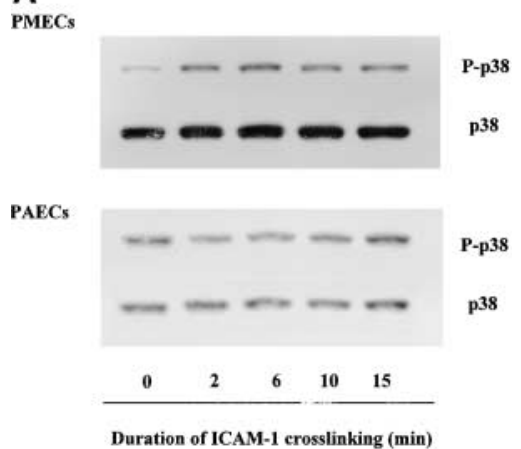

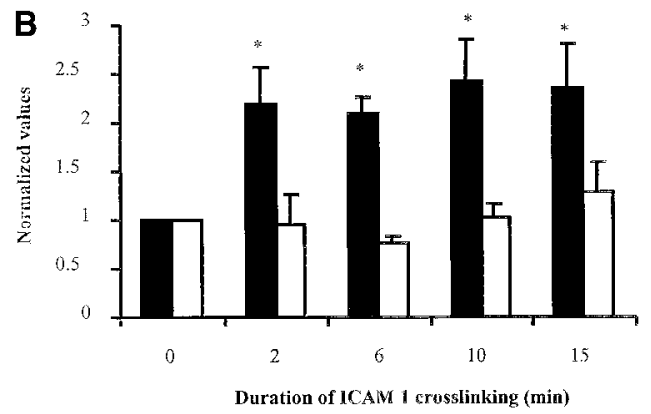

Duration of ICAM 1 cresslinking (min)

Figure 7. ICAM-1 cross-linking induced p38 MAPK activation in PMECs but not in PAECs. TNF- $\alpha$-treated ECs were incubated with $15 \mu \mathrm{g} / \mathrm{ml}$ anti-ICAM-1 antibody or control mouse IgG for 30 minutes and washed. The cells were then incubated with the secondary antibody for 2-15 minutes. The phosphorylated p38 MAPK and the total p38 MAPK were detected by immunoblot. $(A)$ A representative immunoblot showing phosphorylation of p38 MAPK induced by ICAM-1 cross-linking in PMECs but not in PAECs. $(B)$ Densitometric analysis of the immunoblots as in $(A)$. Phosphorylated p38 MAPK was normalized by total p38 levels for each sample, and the data were normalized to the baseline values. The data are expressed as mean \pm SEM $(n=3$ or 4$) .{ }^{*} p<0.05$ compared with the baseline values. Closed bars, PMECs; open bars, PAECs.

quent studies compared ICAM-1-dependent signaling events, including ICAM-1 clustering, activation of xanthine oxidase, ROS production, and p38 MAPK activation. These studies showed that the ICAM-1-dependent signaling events required for these cytoskeletal changes were differentially regulated in these two EC types, and the differences in the signaling pathways appeared to lie downstream of ICAM-1 clustering but upstream of ROS production and subsequent p38 MAPK activation. These studies indicate that there appear to be distinct differences in neutrophil-induced cytoskeletal changes in ECs and in the underlying signaling mechanisms between these two cell types.

The increased expression of ICAM-1 induced by TNF- $\alpha$ as well as redistribution of ICAM-1 induced by cross-linking antibodies were similar in these two cell types, suggesting that the differences in cytoskeletal changes observed in PAECs and PMECs were not due to differences in ICAM-1 expression or in the signaling pathways leading to ICAM-1 redistribution. The signaling events resulting in ICAM-1 clustering in pulmonary ECs are not fully understood. In human umbilical vein endothelial cells, ICAM-1 clustering and redistribution induced by crosslinking antibodies requires activation of Rho (11).

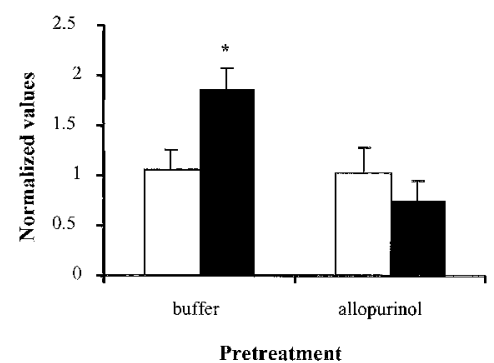

Figure 8. Activation of $\mathrm{p} 38$ MAPK induced by ICAM-1 cross-linking was inhibited by treatment with allopurinol, a xanthine oxidase inhibitor. TNF- $\alpha$-treated PMECs were pretreated with $0.3 \mathrm{mg} / \mathrm{ml}$ allopurinol or control vehicle for 30 minutes. ICAM-1 cross-linking was performed, and phosphorylated p38 MAPK was determined as described in METHODs. Phosphorylated p38 MAPK was normalized by total p38 levels for each sample, and the data were normalized to the baseline values. The data are expressed as mean $\pm \operatorname{SEM}(n=4$ or 6$)$. ${ }^{*} \mathrm{p}<0.05$ when compared with the baseline values. Open bars, no ICAM-1 cross-linking; closed bars, ICAM-1 cross-linking for 6 minutes.
Examination of downstream signaling events induced by ICAM-1 ligation revealed marked differences in the activity of xanthine oxidase and in neutrophil-induced ROS production between PAECs and PMECs. First, the activity of xanthine oxidase in untreated or TNF- $\alpha$-pretreated PAECs was significantly higher than that in PMECs; yet, the baseline ROS production was not different in these two cell types when measured by DCF fluorescence. Thus, oxidant-generating mechanisms other than xanthine oxidase may contribute more to the baseline oxidant production in PMECs than in PAECs. Our previous studies in human PMECs demonstrate that treatment with allopurinol only partially reduces the baseline DCF fluorescence, suggesting that other organelles such as mitochondria may contribute to the baseline ROS production (1). Alternatively, it is possible that the oxidants may be produced at a sequestered site not accessible by the reduced form of DCF. In addition, antioxidants may scavenge the superoxide before it reacts with the reduced form of DCF. Uric acid, produced together with the superoxide in xanthine oxidase-catalyzed reactions, can scavenge ROS and function as an antioxidant (12). In addition, the availability of substrates for xanthine oxidase may be different in these two cell types. Second, xanthine oxidase activity increased in response to neutrophil adherence for 15 minutes in PMECs but not in PAECs. Neither addition of exogenous leukocyte elastase nor mixing neutrophils with endothelial cell lysates without adhesion caused an increase in xanthine oxidase activity, suggesting that this increase in xanthine oxidase activity was not due to neutrophil-derived elastase cleaving xanthine dehydrogenase to the active xanthine oxidase. Consistent with these observations of xanthine oxidase activity, neutrophil adherence induced a significant increase in ROS production only in rat PMECs and not in PAECs. Neutrophil adherence to human PMECs also induces ROS production that is partly inhibited by allopurinol, a xanthine oxidase inhibitor (1). These studies indicate that these two types of ECs differ in their basal xanthine oxidase activity as well as in their ability to produce ROS after neutrophil adherence.

This increase in ROS production in PMECs and not in PAECs, in turn, resulted in the activation of p38 MAPK in PMECs and not in PAECs. Indeed, xanthine oxidase-dependent activation of p38 MAPK in response to ICAM-1 cross-linking was observed only in PMECs and not in PAECs. These data demonstrated that the signaling pathways leading to ROS production and subsequent p38 MAPK activation in response to 
ICAM-1 cross-linking in PMECs were absent in PAECs. The absence of these ICAM-1-initiated signaling pathways in PAECs may likely account for the absence of cytoskeletal rearrangements in these cells in response to neutrophil adherence.

This study, together with our previous studies on human PMECs, supports a role for xanthine oxidase in ICAM-1initiated signaling events and cytoskeletal changes in PMECs during neutrophil adherence. In human PMECs, neutrophil adherence-induced ROS production in ECs is inhibited by an anti-ICAM-1 antibody and is partly inhibited by allopurinol, a xanthine oxidase inhibitor (1). In addition, neutrophil adherence or cross-linking ICAM-1-induced changes in the PMEC cytoskeleton are inhibited by allopurinol as well as by scavengers of ROS (1). This study presents direct evidence for the activation of xanthine oxidase in response to neutrophil adherence in PMECs but not in PAECs. Moreover, allopurinol inhibits activation of p38 MAPK in response to ICAM-1 ligation in human and rat PMECs, and activation of p38 in ECs is required for the changes in actin cytoskeleton in PMECs as well as for neutrophil migration toward EC borders (3). All these data suggest that activation of xanthine oxidase occurs in response to ICAM-1 ligation and is required for downstream events, including p38 activation and actin cytoskeletal changes.

The present study demonstrated that ECs derived from different vascular beds had distinct characteristics, as also revealed by studies from many other laboratories. We found that PAECs had much higher xanthine oxidase activity and that neutrophilinduced ROS production, p38 activation, and EC stiffening occurred only in PMECs and not in PAECs. In addition, Murphy and colleagues (13) showed that PMECs are more susceptible to injury induced by the adhesion of phorbol 12-myristate 13-acetate (PMA)-activated neutrophils than are PAECs. Moreover, Kelly and coworkers (6) demonstrated that thapsigargin, an agent that activates store-operated $\mathrm{Ca}^{2+}$ entry, or calcium ionophores induce increases in permeability in cultured rat PAECs and not in PMECs, despite similar $\mathrm{Ca}^{2+}$ changes in both cell types. In vivo, administration of thapsigargin to isolated lungs induces edema accumulation and gap formation in vessels whose diameters are greater than $100 \mu \mathrm{m}$ but not around microvessels (14). Taken together, these studies support the hypothesis that the differences observed in isolated ECs from different vascular beds in vitro may reflect their physiologic responses and functions in vivo.

In summary, this study demonstrated that neutrophil adherence induced EC stiffening and an increase in F-actin formation only in PMECs, the site of neutrophil emigration during pulmonary inflammation, and not in PAECs. These differences were due to the differences in ICAM-1-dependent signaling events in these two cell types. Understanding these differences may help in the interpretation of the physiologic functions of these changes in regulating neutrophil transmigration during inflammatory responses in the lung. Our previous studies demonstrate that inhibition of neutrophil-induced signaling events in ECs prevents neutrophil migration toward EC borders, the site of most neutrophil transmigration during pulmonary inflammation $(3,15)$. Because neutrophil sequestration and emigration underlie many acute pulmonary inflammatory responses including pneumonia and acute respiratory distress syndrome (reviewed by Lee and Downey [10]), understanding the molecular and cellular mechanisms regulating neutrophil emigration will help better understand how to enhance this process when it is beneficial to the host and to prevent it when it is harmful. Ultimately, this may help identify novel targets for therapeutic interventions.

Acknowledgment: The studies examining EC stiffness were performed at the Physiology Program, Harvard School of Public Health, Boston, MA.

\section{References}

1. Wang Q, Doerschuk CM. Neutrophil-induced changes in the biomechanical properties of endothelial cells: the roles of ICAM-1 and reactive oxygen species. J Immunol 2000;164:6487-6494.

2. Wang Q, Chiang ET, Lim M, Lai J, Rogers R, Janmey PA, Shepro D, Doerschuk CM. Changes in the biomechanical properties of neutrophils and endothelial cells during adhesion. Blood 2001;97:660-668.

3. Wang Q, Doerschuk CM. p38 MAP kinase mediates cytoskeletal remodeling in pulmonary microvascular endothelial cells upon ICAM-1 ligation. J Immunol 2001;166:6877-6884.

4. Moore TM, Chetham PM, Kelly JJ, Stevens T. Signal transduction and regulation of lung endothelial cell permeability. Interaction between calcium and cAMP. Am J Physiol 1998;275:L203-L222.

5. Diwan AH, Thompson WJ, Lee AK, Strada SJ. Cyclic GMP-dependent protein kinase activity in rat pulmonary microvascular endothelial cells. Biochem Biophys Res Commun 1994;202:728-735.

6. Kelly JJ, Moore TM, Basal P, Diwan AH, Stevens T, Thompson WJ. Pulmonary microvascular and macrovascular endothelial cells: differential regulation of $\mathrm{Ca}^{2+}$ and permeability. Am J Physiol 1998;274: L810-L819.

7. Stevens T, Creighton J, Thompson WJ. Control of cAMP in lung endothelial cell phenotypes. Implications for control of barrier function. Am J Physiol 1999;277:L119-L126.

8. Ozer N, Muftuoglu M, Ogus IH. A simple and sensitive method for the activity staining of xanthine oxidase. J Biochem Biophys Methods 1998; 36:95-100.

9. Lawson C, Ainsworth M, Yacoub M, Rose M. Ligation of ICAM-1 on endothelial cells leads to expression of VCAM-1 via a nuclear factorкB-independent mechanism. I Immunol 1999;162:2990-2996.

10. Lee WL, Downey GP. Leukocyte elastase. Am J Respir Crit Care Med 2001;164:896-904.

11. Wojciak-Stothard B, Williams L, Ridley AJ. Monocyte adhesion and spreading on human endothelial cells is dependent on Rho-regulated receptor clustering. J Cell Biol 1999;145:1293-1307.

12. Sanders KA, Huecksteadt T, Xu P, Sturrock AB, Hoidal JR. Regulation of oxidant production in acute lung injury. Chest 1999;116:56S-61S.

13. Murphy HS, Bakopoulos N, Dame MK, Varani J, Ward PA. Heterogeneity of vascular endothelial cells: differences in susceptibility to neutrophil-mediated injury. Microvasc Res 1998;56:203-211.

14. Chetham PM, Basal P, Bridges JP, Moore TM, Stevens T. Segmental regulation of pulmonary vascular permeability by store-operated $\mathrm{Ca}^{2+}$ entry. Am J Physiol 1999;276:L41-L50.

15. Walker DC, Behzad AR, Chu F. Neutrophil migration through preexisting holes in the basal laminae of alveolar capillaries and epithelium during streptococcal pneumonia. Microvasc Res 1995;50:397-416. 